Research Article

\title{
Solvability for a Fully Elastic Beam Equation with Left-End Fixed and Right-End Simply Supported
}

\author{
Mei Wei (i) and Yongxiang $\mathrm{Li}$ (iD) \\ Department of Mathematics, Northwest Normal University, Lanzhou 730070, China \\ Correspondence should be addressed to Mei Wei; nwnuweimei@126.com and Yongxiang Li; liyxnwnu@163.com
}

Received 1 March 2021; Accepted 22 May 2021; Published 8 June 2021

Academic Editor: Jing-Feng Tian

Copyright (๑) 2021 Mei Wei and Yongxiang Li. This is an open access article distributed under the Creative Commons Attribution License, which permits unrestricted use, distribution, and reproduction in any medium, provided the original work is properly cited.

The aim of the present paper is to consider a fully elastic beam equation with left-end fixed and right-end simply supported, i.e., $\left\{\begin{array}{l}u^{(4)}(t)=f\left(t, u(t), u^{\prime}(t), u^{\prime \prime}(t), u^{\prime \prime \prime}(t)\right), t \in[0,1] \\ u(0)=u^{\prime}(0)=u(1)=u^{\prime \prime}(1)=0\end{array}\right.$ point theorem of the completely continuous operator, the existence and uniqueness of solutions are obtained under the conditions that the nonlinear function satisfies the linear growth and superlinear growth. For the case of superlinear growth, a Nagumo-type condition is introduced to limit that $f\left(t, x_{0}, x_{1}, x_{2}, x_{3}\right)$ is quadratical growth on $x_{3}$ at most.

\section{Introduction}

This paper focuses on the existence and uniqueness of solutions for the boundary value problems (BVP) of the fourth-order ordinary differential equation:

$$
\left\{\begin{array}{l}
u^{(4)}(t)=f\left(t, u(t), u^{\prime}(t), u^{\prime \prime}(t), u^{\prime \prime \prime}(t)\right), \quad t \in[0,1], \\
u(0)=u^{\prime}(0)=u(1)=u^{\prime \prime}(1)=0,
\end{array}\right.
$$

where $f:[0,1] \times \mathbb{R}^{4} \longrightarrow \mathbb{R}$ is continuous and involves all derivatives below the fourth order of unknown functions. The problem simulates the deformation of an elastic beam with the left-end fixed and the right simply supported.

The boundary value problems for the nonlinear fourthorder differential equations are the mathematical models used to characterize the deflection of elastic beams under external forces. Elastic beams are one of the most basic structures in architecture and engineering, and some different boundary conditions are derived due to the diversity of stress states on its two ends.

In the past few years, owing to its actual mathematical model and wide application background, the research on nonlinear fourth-order two-point BVP has been very active. Its solvability has attracted the close attention of many scholars, and some profound results have been obtained through various nonlinear analysis methods and techniques (See [1-22] and its references).

There are many results on the solvability for the special cases of BVP (1) whose nonlinear term $f$ is independent of the third-order derivative term of $u$ (see $[2,8,10,15]$ ). However, only few articles have studied the existence of solutions for BVP (1). It is worth noting that, in [12], Yao proved the existence of the solutions for BVP (1) by calculating the maximum value of Green function and its partial derivatives as well as constructing height functions of $f$ on bounded sets. In addition, the research on its solvability under some excellent growth conditions, especially the superlinear growth conditions, is even more rare.

In the mechanical analysis of beams, the physical meaning of the derivatives $u^{\prime}(t), u^{\prime \prime}(t), u^{\prime \prime \prime}(t)$, and $u^{(4)}$ of $u(t)$ are slope, bending moment, shear force, and load density, respectively (see $[1,2,17-19]$ ). The existence of slope, bending moment, shear force, and load density is undoubtedly very beneficial to the complete stress analysis of beams. Nevertheless, the dependence of $f$ on the third derivative $u$ 'I" increases the difficulty for our study, but this is also a fundamental difference from the previous problems. In recent years, the research on the solvability of the elastic 
beam equation that $f$ involves all lower-order derivatives of deformation function $u$ has become a hot topic (see $[5,6,11-22])$. For example, the elastic beam equation whose both ends are simply supported (see $[14,16,20])$ :

$$
\left\{\begin{array}{l}
u^{(4)}(t)=f\left(t, u(t), u^{\prime}(t), u^{\prime \prime}(t), u^{\prime \prime \prime}(t)\right), \quad t \in[0,1] \\
u(0)=u^{\prime \prime}(0)=u(1)=u^{\prime \prime}(1)=0
\end{array}\right.
$$

and one end is simply supported, and the other end is sliding clamped (see $[5,11,22])$ :

$$
\left\{\begin{array}{l}
u^{(4)}(t)=f\left(t, u(t), u^{\prime}(t), u^{\prime \prime}(t), u^{\prime \prime \prime}(t)\right), \quad t \in[0,1] \\
u(0)=u^{\prime \prime}(0)=u^{\prime}(1)=u^{\prime \prime \prime}(1)=0
\end{array}\right.
$$

and one end is fixed, and the other end is free (see $[13,17-19,21])$ :

$$
\left\{\begin{array}{l}
u^{(4)}(t)=f\left(t, u(t), u^{\prime}(t), u^{\prime \prime}(t), u^{\prime \prime \prime}(t)\right), \quad t \in[0,1] \\
u(0)=u^{\prime}(0)=u^{\prime \prime}(1)=u^{\prime \prime \prime}(1)=0
\end{array}\right.
$$

The solvability of equations (2)-(4) has been studied by various nonlinear functional methods, including fixed point index theory in cone, fixed point theorem, lower and upper solutions' method, topological degree theory, and variational method. In particular, in [16], Li and Liang researched the existence and uniqueness of solutions for BVP (2) under the condition that $f\left(t, x_{0}, x_{1}, x_{2}, x_{3}\right)$ is linear growth; in [19], by supplementing the Nagumo-type condition to limit the growth of $f$ on $x_{3}, \mathrm{Li}$ and Chen obtained the existence and uniqueness of solutions for BVP (4) under the condition that $f$ satisfies one-side superlinear growth.

Inspired by the literature listed above, in the present article, we discuss the existence and uniqueness of solutions for BVP (1) under the linear growth and one-side superlinear growth condition. The results of existence and uniqueness under the linear growth are presented in Section 3, and the existence results under the one-side superlinear growth are presented in Section 4. It should be noted that, in this paper, the estimation of the maximum value of Green function and its partial derivatives is no longer needed. In addition, with the help of efficient norm estimation and Leray-Schauder fixed point theorem of completely continuous operator, our discussion is carried out in the whole workspace without the restrictions of boundedness of $u$ and its derivatives. Therefore, our conclusions greatly improve and generalize the case of the bounded domain in the existing literature, which is new and significant. In order to prove the conclusion, we introduce some necessary properties of the solutions for the corresponding linear equation in the following section.

\section{Preliminaries}

Let $I=[0,1], n \in \mathbb{N}$. We introduce the following common spaces on $I$ :

(i) $C(I)$ be the continuous function space with the norm $\|u\|_{C}=\max _{t \in I}|u(t)|$

(ii) $L^{2}(I)$ denotes the Hilbert space formed by inner product $(u, v)=\int_{0}^{1} u(t) v(t) \mathrm{d} t$ of all Lebesgue square integrable functions on $I$, and its inner product norm is $\|u\|_{2}=\left(\int_{0}^{1}|u(t)|^{2} \mathrm{~d} t\right)^{(1 / 2)}$

(iii) $C^{n}(I)$ be the $n$-order continuous differentiable function space with the norm $\|u\|_{C^{n}}=\max \left\{\|u\|_{C},\left\|u^{\prime}\right\|_{C}, \ldots,\left\|u^{(n)}\right\|_{C}\right\}$

(iv) $H^{n}(I)$ be the Sobolev space constituted by norm $\|u\|_{n, 2}=\left(\sum_{i=0}^{n}\left\|u^{(i)}\right\|_{2}^{2}\right)^{(1 / 2)}, u \in H^{n}(I)$ shows that $u \in C^{n-1}(I), u^{(n-1)}(t)$ is absolutely continuous on $I$, and $u^{(n)} \in L^{2}(I)$

Firstly, we consider the following linear boundary value problem (LBVP) corresponding to BVP (1):

$$
\left\{\begin{array}{l}
u^{(4)}(t)=h(t), \quad t \in I, \\
u(0)=u^{\prime}(0)=u(1)=u^{\prime \prime}(1)=0 .
\end{array}\right.
$$

Lemma 1. For $h \in L^{2}(I), L B V P$ (5) exists a unique solution $u:=S h \in H^{4}(I)$, and the solution operator $S: L^{2}(I) \longrightarrow H^{4}(I)$ is a bounded linear operator. Furthermore, $S: L^{2}(I) \longrightarrow H^{3}(I)$ is completely continuous.

Proof. For each $h \in L^{2}(I)$, one can easily test that

$$
u(t)=\int_{0}^{1} G(t, s) h(s) \mathrm{d} s:=S h(t) \in H^{4}(I), \quad t \in I,
$$

is a solution of LBVP (5), where

$$
G(t, s)=\frac{1}{12} \begin{cases}t^{2}(1-s)\left[3(1-t)-(1-s)^{2}(3-t)\right], & 0 \leq t \leq s \leq 1, \\ s^{2}(1-t)\left[3(1-s)-(1-t)^{2}(3-s)\right], & 0 \leq s \leq t \leq 1,\end{cases}
$$

is the Green function corresponding to the homogeneous linear problem, and it is obvious that $G(t, s) \geq 0, t, s \in I$. Through specific calculations, we can get

$$
0 \leq \int_{0}^{1} G(t, s) \mathrm{d} s=\frac{1}{48} t^{2}(1-t)(3-2 t)<1 .
$$


Therefore, by applying the contraction mapping principle to the solution operator $S$ and combining with (6), it is easy to prove that $S$ has only one fixed point on $L^{2}(I)$, that is, $u$ shown in (6) is the unique solution of LBVP (5). When $h \in C(I), u(t):=S h \in C^{4}(I)$ is a unique classical solution of LBVP (5). It is clear that $G(t, s)$ is continuous; then, according to (6), $S: L^{2}(I) \longrightarrow H^{4}(I)$ is a bounded linear operator.

Furthermore, $S: L^{2}(I) \longrightarrow H^{3}(I)$ is obviously completely continuous since the Sobolev embedding $H^{4}(I) \hookrightarrow C^{3}(I)$ is compact and the embedding $C^{3}(I) \hookrightarrow H^{3}(I)$ is continuous.

The proof is finished.
Lemma 2. The unique solution $u$ of LBVP (5) has the following properties:
(a) $u^{\prime \prime}(0) u^{\prime \prime \prime}(0) \leq 0$
(b) $u^{\prime \prime \prime}(0) u^{\prime \prime \prime}(1) \leq 0$
(c) $\left\|u^{(i)}\right\|_{2} \leq(1 / \sqrt{2})\left\|u^{(i+1)}\right\|_{2}, i=0,1,2$, and 3

Proof

(a) With a simple calculation, we get

$$
\begin{aligned}
& \frac{\partial^{2}}{\partial t^{2}} G(t, s)=\frac{1}{2} \begin{cases}(1-s)\left[1-3 t-(1-t)(1-s)^{2}\right], & 0 \leq t \leq s \leq 1, \\
s^{2}(1-t)(s-3), & 0 \leq s \leq t \leq 1,\end{cases} \\
& \frac{\partial^{3}}{\partial t^{3}} G(t, s)=\frac{1}{2} \begin{cases}(1-s)\left(s^{2}-2 s-2\right), & 0 \leq t<s \leq 1, \\
s^{2}(3-s), & 0 \leq s<t \leq 1 .\end{cases}
\end{aligned}
$$

Then, we can verify that, for given $h \in C(I)$,

$$
\begin{aligned}
& u^{\prime \prime}(0)=\frac{1}{2} \int_{0}^{1}(1-s)\left(1-(1-s)^{2}\right) h(s) \mathrm{d} s, \\
& u^{\prime \prime \prime}(0)=\frac{1}{2} \int_{0}^{1}(1-s)\left((s-1)^{2}-3\right) h(s) \mathrm{d} s .
\end{aligned}
$$

Since for every $s \in I$,

$$
\begin{aligned}
& (1-s)\left(1-(1-s)^{2}\right) \geq 0, \\
& (1-s)\left((s-1)^{2}-3\right) \leq 0,
\end{aligned}
$$

and thus, $u^{\prime \prime}(0) u^{\prime \prime \prime}(0) \leq 0$.

(b) By (10), we can check that, for given $h \in C(I)$,

$$
u^{\prime \prime \prime}(1)=\frac{1}{2} \int_{0}^{1} s^{2}(3-s) h(s) \mathrm{d} s .
$$

Since for every $s \in I$,

$$
s^{2}(3-s) \geq 0,
$$

and hence, combining with (11) and (12), $u^{\prime \prime \prime}(0) u^{\prime \prime \prime}(1) \leq 0$.

(c) Considering the boundary condition of BVP (5) and using the Hölder inequality, we can obtain that, for every $t \in I$,

$$
\begin{aligned}
|u(t)| & =\left|\int_{0}^{t} u^{\prime}(s) \mathrm{d} s\right| \leq \int_{0}^{t}\left|u^{\prime}(s)\right| \mathrm{d} s \\
& \leq\left(\int_{0}^{t}\left|u^{\prime}(s)\right|^{2} \mathrm{~d} s\right)^{(1 / 2)} \cdot\left(\int_{0}^{t} 1 \mathrm{~d} s\right)^{(1 / 2)} \\
& \leq t^{(1 / 2)}\left\|u^{\prime}\right\|_{2},
\end{aligned}
$$

and then,

$$
\|u\|_{2}=\left(\int_{0}^{1}|u(t)|^{2} \mathrm{~d} t\right)^{(1 / 2)} \leq\left(\int_{0}^{1} t\left\|u^{\prime}\right\|_{2}^{2} \mathrm{~d} t\right)^{(1 / 2)} \leq \frac{1}{\sqrt{2}}\left\|u^{\prime}\right\|_{2} .
$$

In the same way, we have

$$
\begin{aligned}
& \left|u^{\prime}(t)\right|=\left|\int_{0}^{t} u^{\prime \prime}(s) \mathrm{d} s\right| \leq t^{(1 / 2)}\left\|u^{\prime \prime}\right\|_{2}, \\
& \left|u^{\prime \prime}(t)\right|=\left|-\int_{t}^{1} u^{\prime \prime \prime}(s) \mathrm{d} s\right| \leq(1-t)^{(1 / 2)}\left\|u^{\prime \prime \prime}\right\|_{2} .
\end{aligned}
$$

Therefore,

$$
\begin{aligned}
\left\|u^{\prime}\right\|_{2} & \leq \frac{1}{\sqrt{2}}\left\|u^{\prime \prime}\right\|_{2}, \\
\left\|u^{\prime \prime}\right\|_{2} & \leq \frac{1}{\sqrt{2}}\left\|u^{\prime \prime \prime}\right\|_{2} .
\end{aligned}
$$


According to the conclusion of (b) and the continuity of $u^{\prime \prime \prime}(t)$, there exists $t_{0} \in[0,1]$ such that $u^{\prime \prime \prime}\left(t_{0}\right)=0$. Thus,

$$
u^{\prime \prime \prime}(t)= \begin{cases}\int_{t_{0}}^{t} u^{(4)}(s) \mathrm{d} s, & t \in\left[t_{0}, 1\right], \\ -\int_{t}^{t_{0}} u^{(4)}(s) \mathrm{d} s, & t \in\left[0, t_{0}\right] .\end{cases}
$$

We can find out in just the same way that

$$
\left\|u^{\prime \prime \prime}\right\|_{2} \leq \frac{1}{\sqrt{2}}\left\|u^{(4)}\right\|_{2} \text {. }
$$

Those show that (c) is valid.

At this point, the proof is finished.

Finally, we introduce the famous Leray-Schsuder fixed point theorem, which will be used to establish our main theorems.

Lemma 3 (see [7]). Let $X$ be a Banach space, and $T: X \longrightarrow X$ is a completely continuous operator. If the solution set of the homotopy family equation

$$
x=\lambda T x, \quad 0<\lambda<1,
$$

is bounded in $X$, then there exists a fixed point of $T$ in $X$.

\section{Solvability under Linear Growth}

Theorem 1. Suppose that $\quad f \in C\left(I \times \mathbb{R}^{4}, \mathbb{R}\right)$ satisfies the following:

(H1) there exist constants $\tilde{a}>0$ and $a_{0}, a_{1}, a_{2}$, and $a_{3} \geq 0$ with $\left(a_{0} / 4\right)+\left(a_{1} / 2 \sqrt{2}\right)+\left(a_{2} / 2\right)+\left(a_{3} / \sqrt{2}\right)<1$ such that, for every $\left(t, x_{0}, x_{1}, x_{2}, x_{3}\right) \in I \times \mathbb{R}^{4}$,

$$
\left|f\left(t, x_{0}, x_{1}, x_{2}, x_{3}\right)\right| \leq a_{0}\left|x_{0}\right|+a_{1}\left|x_{1}\right|+a_{2}\left|x_{2}\right|+a_{3}\left|x_{3}\right|+\tilde{a} .
$$

Then, BVP (1) has at least one solution.

Proof. Define operator $F: H^{3}(I) \longrightarrow L^{2}(I)$ by

$$
F(u)(t)=f\left(t, u(t), u^{\prime}(t), u^{\prime \prime}(t), u^{\prime \prime \prime}(t)\right), \quad t \in I,
$$

and then, $F$ is continuous and maps bounded sets of $H^{3}(I)$ into bounded sets of $L^{2}(I)$. According to the definition of $S$ defined in Lemma 1, the solution of BVP (1) is equivalent to the fixed point of the composition operator:

$$
T:=S \circ F \text {. }
$$

By Lemma $1, T: H^{3}(I) \longrightarrow H^{3}(I)$ is completely continuous. Now, we apply Lemma 3 to verify that $T$ has a fixed point.

Consider the homotopy family equation

$$
u=\lambda T u, \quad 0<\lambda<1 .
$$

Let $u \in H^{3}(I)$ be the solution of equation (25) corresponding to $\lambda \in(0,1)$; then, $u=\lambda T u=\lambda S F(u)=S(\lambda F(u))$ denotes $h=\lambda F(u)$, and then, $u=S h \in H^{4}(I)$ is the unique solution of LBVP (5), so it satisfies

$$
\left\{\begin{array}{l}
u^{(4)}(t)=\lambda f\left(t, u(t), u^{\prime}(t), u^{\prime \prime}(t), u^{\prime \prime \prime}(t)\right), \quad t \in I, \\
u(0)=u^{\prime}(0)=u(1)=u^{\prime \prime}(1)=0 .
\end{array}\right.
$$

Taking $\|\cdot\|_{2}$ norm for both ends of BVP (26), together with $(H 1)$ and Lemma 2 (c), we can obtain

$$
\begin{aligned}
\left\|u^{(4)}\right\|_{2} & =\lambda\|F(u)\|_{2} \leq a_{0}\|u\|_{2}+a_{1}\left\|u^{\prime}\right\|_{2}+a_{2}\left\|u^{\prime \prime}\right\|_{2}+a_{3}\left\|u^{\prime \prime \prime}\right\|_{2}+\tilde{a} \\
& \leq\left(\frac{a_{0}}{4}+\frac{a_{1}}{2 \sqrt{2}}+\frac{a_{2}}{2}+\frac{a_{3}}{\sqrt{2}}\right)\left\|u^{(4)}\right\|_{2}+\tilde{a} .
\end{aligned}
$$

Hence,

$$
\left\|u^{(4)}\right\|_{2} \leq \frac{\tilde{a}}{1-\left(\left(a_{0} / 4\right)+\left(a_{1} / 2 \sqrt{2}\right)+\left(a_{2} / 2\right)+\left(a_{3} / \sqrt{2}\right)\right)}:=C_{0} .
$$

Consequently, combining with Lemma 2 (c),

$$
\begin{aligned}
\|u\|_{3,2} & =\sqrt{\|u\|_{2}^{2}+\left\|u^{\prime}\right\|_{2}^{2}+\left\|u^{\prime \prime}\right\|_{2}^{2}+\left\|u^{\prime \prime \prime}\right\|_{2}^{2}} \\
& \leq \sqrt{\frac{1}{16}+\frac{1}{8}+\frac{1}{4}+\frac{1}{2}}\left\|u^{(4)}\right\|_{2} \\
& <\left\|u^{(4)}\right\|_{2} \leq C_{0} .
\end{aligned}
$$

That is, the solutions' set of equation (25) is bounded in $H^{3}(I)$. Then, in accordance with Lemma 3, there exists $u_{0} \in H^{3}(I)$ is the fixed point of $T$, which is the solution of BVP (1).

The proof of Theorem 1 is completed.

Next, we establish the uniqueness result for BVP (1).

Theorem 2. Suppose that $f \in C\left(I \times \mathbb{R}^{4}, \mathbb{R}\right)$ satisfies the following:

(H2) there exist constants $a_{0}, a_{1}, a_{2}$ and $a_{3} \geq 0$ with $\left(a_{0} / 4\right)+\left(a_{1} / 2 \sqrt{2}\right)+\left(a_{2} / 2\right)+\left(a_{3} / \sqrt{2}\right)<1$ such that, for every $t \in I$ and $x_{i}$ and $y_{i} \in \mathbb{R}, i=0,1,2$, and 3 ,

$$
\left|f\left(t, x_{0}, x_{1}, x_{2}, x_{3}\right)-f\left(t, y_{0}, y_{1}, y_{2}, y_{3}\right)\right| \leq \sum_{i=0}^{3} a_{i}\left|x_{i}-y_{i}\right|
$$

Then, BVP (1) has a unique solution.

Proof. Let $\tilde{a}=\max _{t \in I}|f(t, 0,0,0,0)|+1$. Then, from (H2), it can be clearly deduced that $(H 1)$ is valid. Therefore, by Theorem 1, BVP (1) has at least one solution. Now, we just need to prove the uniqueness.

Let $u_{1}$ and $u_{2}$ be the solutions of BVP (1), then $u_{1}=S\left(F\left(u_{1}\right), u_{2}=S\left(F\left(u_{2}\right)\right)\right.$. Denote $u=u_{2}-u_{1}=S$ $\left(F\left(u_{2}\right)-F\left(u_{1}\right)\right)$, thus $u$ is the unique solution of LBVP (5) for $h=F\left(u_{2}\right)-F\left(u_{1}\right)$. According to (H2) and Lemma 2 (c), 


$$
\begin{aligned}
\left\|u^{(4)}\right\|_{2} & =\left\|F\left(u_{2}\right)-F\left(u_{1}\right)\right\|_{2} \\
& \leq a_{0}\left\|u_{2}-u_{1}\right\|_{2}+a_{1}\left\|u_{2}^{\prime}-u_{1}^{\prime}\right\|_{2}+a_{2}\left\|u_{2}^{\prime \prime}-u_{1}^{\prime \prime}\right\|_{2}+a_{3}\left\|u_{2}^{\prime \prime}-u_{1}^{\prime \prime}\right\|_{2} \\
& =a_{0}\|u\|_{2}+a_{1}\left\|u^{\prime}\right\|_{2}+a_{2}\left\|u^{\prime \prime}\right\|_{2}+a_{3}\left\|u^{\prime \prime \prime}\right\|_{2} \\
& \leq\left(\frac{a_{0}}{4}+\frac{a_{1}}{2 \sqrt{2}}+\frac{a_{2}}{2}+\frac{a_{3}}{\sqrt{2}}\right)\left\|u^{(4)}\right\|_{2} .
\end{aligned}
$$

Since $\left(a_{0} / 4\right)+\left(a_{1} / 2 \sqrt{2}\right)+\left(a_{2} / 2\right)+\left(a_{3} / \sqrt{2}\right)<1, \quad$ it shows that $\left\|u^{(4)}\right\|_{2} \leq 0$; hence, by (29), $\|u\|_{3,2} \leq 0$, that is, $u_{1}=u_{2}$. Consequently, there is only one solution for BVP (1).

The proof of the theorem is completed.

If the partial derivatives $f_{x_{0}}, f_{x_{1}}, f_{x_{2}}$, and $f_{x_{3}}$ of $f\left(t, x_{0}, x_{1}, x_{2}, x_{3}\right)$ exist, then we can obtain the following conclusion by means of the differential mean value theorem.

Corollary 1. Suppose that $f \in C\left(I \times \mathbb{R}^{4}, \mathbb{R}\right)$. If the partial derivatives $f_{x_{0}}, f_{x_{1}}, f_{x_{2}}$, and $f_{x_{3}}$ of $f\left(t, x_{0}, x_{1}, x_{2}, x_{3}\right)$ on $x_{0}, x_{1}, x_{2}$, and $x_{3}$ exist and satisfy,

(H3) there exist constants $a_{0}, a_{1}, a_{2}$, and $a_{3} \geq 0$ with $\left(a_{0} / 4\right)+\left(a_{1} / 2 \sqrt{2}\right)+\left(a_{2} / 2\right)+\left(a_{3} / \sqrt{2}\right)<1$ such that, for every $\left(t, x_{0}, x_{1}, x_{2}, x_{3}\right) \in I \times \mathbb{R}^{4}$,

$$
\left|f_{x_{i}}\left(t, x_{0}, x_{1}, x_{2}, x_{3}\right)\right| \leq a_{i}, i=0,1,2 \text {, and } 3 \text {. }
$$

Then, BVP (1) has a unique solution.

Example 1. Consider the fully nonlinear BVP

$$
\left\{\begin{array}{l}
u^{(4)}(t)=2 u^{(1 / 3)}(t)+t^{2}\left(u^{\prime}(t)\right)^{(1 / 3)}+\sin t\left(u^{\prime \prime}(t)\right)^{(1 / 3)}+e^{-t}\left|u^{\prime \prime \prime}(t)\right|^{(1 / 2)}+e^{t}, \quad t \in I, \\
u(0)=u^{\prime}(0)=u(1)=u^{\prime \prime}(1)=0 .
\end{array}\right.
$$

The nonlinear terms

$$
\begin{aligned}
f\left(t, x_{0}, x_{1}, x_{2}, x_{3}\right)= & 2 x_{0}^{(1 / 3)}+t^{2} \cdot x_{1}^{(1 / 3)}+\sin t \cdot x_{2}^{(1 / 3)} \\
& +e^{-t}\left|x_{3}\right|^{(1 / 2)}+e^{t} .
\end{aligned}
$$

Since

$$
\begin{aligned}
& |x|^{(1 / 2)} \leq \frac{1}{4}|x|+1, \\
& |x|^{(1 / 3)} \leq \frac{1}{4}|x|+\frac{5}{4} .
\end{aligned}
$$

Therefore,

$$
f\left(t, x_{0}, x_{1}, x_{2}, x_{3}\right) \leq \frac{1}{2}\left|x_{0}\right|+\frac{1}{4}\left|x_{1}\right|+\frac{1}{4}\left|x_{2}\right|+\frac{1}{4}\left|x_{3}\right|+6+e .
$$

Set $a_{0}=1 / 2, a_{1}=a_{2}=a_{3}=1 / 4$, and $\tilde{a}=6+e$, then $\left(a_{0} / 4\right)+\left(a_{1} / 2 \sqrt{2}\right)+\left(a_{2} / 2\right)+\left(a_{3} / \sqrt{2}\right)=(1+3 \sqrt{2}) / 8 \sqrt{2}$ $<1$, which implies that $f$ satisfies the condition $(H 1)$; then, by Theorem 1, BVP (33) has at least one solution.

Remark 1. In fact, for any $t \in I, \widetilde{\alpha}(t), \alpha_{i}(t)$ $\in C(I)$, and $\beta_{i} \in(0,1), i=0,1,2$, and 3 , let

$$
f\left(t, x_{0}, x_{1}, x_{2}, x_{3}\right)=\sum_{i=0}^{3} \alpha_{i}(t)\left|x_{i}\right|^{\beta_{i}}+\widetilde{\alpha}(t),
$$

then corresponding BVP (1) has at least one solution.

\section{Solvability under Superlinear Growth}

In this section, in order to facilitate the establishment of the theorem, we need a Nagumo-type condition to limit the growth of $f$ on $x_{3}$ and an important Lemma for the Nagumo-type condition,

$\left(H^{*}\right)$ for any $M>0$, there is a function $H_{M}(\rho) \in C\left(\mathbb{R}^{+}, \mathbb{R}^{+} \backslash\{0\}\right)$ with

$$
\int_{0}^{+\infty} \frac{\rho}{H_{M}(\rho)} \mathrm{d} \rho=+\infty,
$$

such that, for every $\left(t, x_{0}, x_{1}, x_{2}, x_{3}\right) \in I \times[-M, M]^{3} \times \mathbb{R}$,

$$
\left|f\left(t, x_{0}, x_{1}, x_{2}, x_{3}\right)\right| \leq H_{M}\left(\left|x_{3}\right|\right) \text {. }
$$

Lemma 4. Suppose that $f \in C\left(I \times \mathbb{R}^{4}, \mathbb{R}\right)$ satisfies $\left(H^{*}\right)$. If there exists $M>0$ such that the solution $u$ of $B V P(1)$ satisfies $\|u\|_{C^{2}} \leq M$, then there exists $M_{1}=M_{1}(M)>0$, such that $\left\|u^{\prime \prime \prime}\right\|_{C} \leq M_{1}$.

Proof. Let $M>0$, then by (38), there is a constant $M_{1}>0$ satisfying

$$
\int_{0}^{M_{1}} \frac{\rho}{H_{M}(\rho)} \mathrm{d} \rho>2 M .
$$

Set $u$ be a solution of BVP (1) that satisfies $\|u\|_{C^{2}} \leq M$; now, we check $\left\|u^{\prime \prime \prime}\right\|_{C} \leq M_{1}$. From Lemma 2 (b), it follows that there exists $t_{0} \in I$, such that $u^{\prime \prime \prime}\left(t_{0}\right)=0$. Suppose $\left\|u^{\prime \prime \prime}\right\|_{C} \neq 0$, that is, $\left\|u^{\prime \prime \prime}\right\|_{C}>0$, then there exists $t_{1} \in I$ such that

$$
\left\|u^{\prime \prime \prime}\right\|_{C}=\max _{t \in I}\left|u^{\prime \prime \prime}(t)\right|=\left|u^{\prime \prime \prime}\left(t_{1}\right)\right|>0 .
$$

Therefore, $t_{0} \neq t_{1}$. Thus, there are four cases:

(1) $u^{\prime \prime \prime}\left(t_{1}\right)>0$ and $t_{0}<t_{1}$

(2) $u^{\prime \prime \prime}\left(t_{1}\right)>0$ and $t_{0}>t_{1}$

(3) $u^{\prime \prime \prime}\left(t_{1}\right)<0$ and $t_{0}<t_{1}$ 
(4) $u^{\prime \prime \prime}\left(t_{1}\right)<0$ and $t_{0}>t_{1}$

For case 1, let (see Figure 1)

$$
t_{2}=\sup \left\{t \in\left[t_{0}, t_{1}\right] \mid u^{\prime \prime \prime}(t)=0\right\}
$$

and according to the definition of supremum and the continuity of $u^{\prime \prime \prime}(t)$, we have $t_{0} \leq t_{2}<t_{1} \leq 1$ and

$$
u^{\prime \prime \prime}\left(t_{2}\right)=0, u^{\prime \prime \prime}(t)>0 \text { and } t \in\left(t_{2}, t_{1}\right] .
$$

Since

$$
|u(t)|,\left|u^{\prime}(t)\right|,\left|u^{\prime \prime}(t)\right| \leq\|u\|_{C^{2}} \leq M,
$$

hence, by BVP (1) and (39),

$$
u^{(4)}(t)=f\left(t, u(t), u^{\prime}(t), u^{\prime \prime}(t), u^{\prime \prime \prime}(t)\right) \leq H_{M}\left(u^{\prime \prime \prime}(t)\right), \quad t \in\left(t_{2}, t_{1}\right] .
$$

Then,

$$
\frac{u^{(4)}(t) u^{\prime \prime \prime}(t)}{H_{M}\left(u^{\prime \prime \prime}(t)\right)} \leq u^{\prime \prime \prime}(t), \quad t \in\left(t_{2}, t_{1}\right]
$$

Taking integral operation on $\left(t_{2}, t_{1}\right]$ for both sides of the inequality, and making variable substitution $\rho=u^{\prime \prime \prime}(t)$ on the left. Then,

$$
\int_{0}^{u^{\prime \prime \prime}\left(t_{1}\right)} \frac{\rho d \rho}{H_{M}\left(u^{\prime \prime \prime}(t)\right)} \leq u^{\prime \prime}\left(t_{1}\right)-u^{\prime \prime}\left(t_{2}\right) \leq 2\left\|u^{\prime \prime}\right\|_{C} \leq 2\|u\|_{C^{2}} \leq 2 M .
$$

Thereby,

$$
\int_{0}^{\left\|u^{\prime \prime \prime}\right\|_{C}} \frac{\rho d \rho}{H_{M}(\rho)+C_{0}} \leq 2 M
$$

Then, based on (40),

$$
\left\|u^{\prime \prime \prime}\right\|_{C} \leq M_{1} .
$$

Similarly, we can discuss cases $2^{\circ}-4^{\circ}$, and the conclusions are the same.

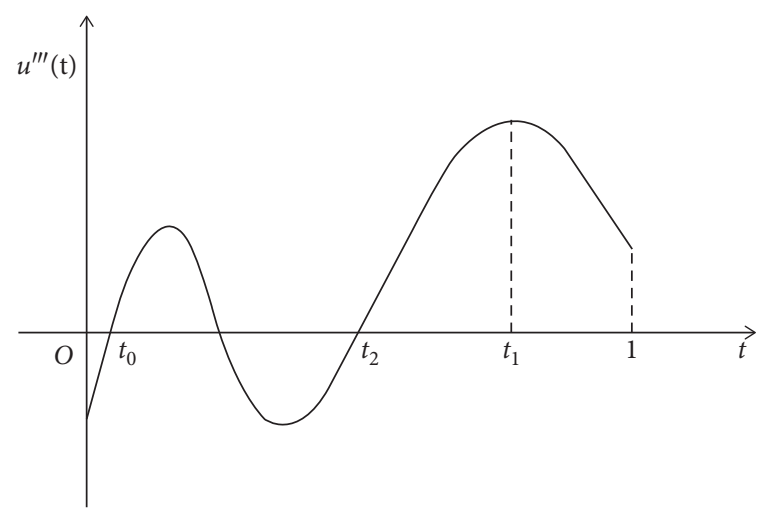

Figure 1: $u^{\prime \prime \prime}\left(t_{1}\right)>0$ and $t_{0}<t_{1}$.

Theorem 3. Suppose that $f \in C\left(I \times \mathbb{R}^{4}, \mathbb{R}\right)$ satisfies the following:

$\left(H^{*}\right)$ and (H4) there exist constants $\tilde{b}>0$ and $b_{0}, b_{1}, b_{2}$, and $b_{3} \geq 0$ with $\left(b_{0} / 8\right)+\left(b_{1} / 4\right)+\left(b_{2} / 2\right)+$ $b_{3}<1$ such that, for every $\left(t, x_{0}, x_{1}, x_{2}, x_{3}\right) \in I \times \mathbb{R}^{4}$,

$-f\left(t, x_{0}, x_{1}, x_{2}, x_{3}\right) x_{2} \leq b_{0} x_{0}^{2}+b_{1} x_{1}^{2}+b_{2} x_{2}^{2}+b_{3} x_{3}^{2}+\tilde{b}$.

Then, BVP (1) has at least one solution.

Proof. Define operator $F: C^{3}(I) \longrightarrow C(I)$ by (23). According to Lemma 1, it is evident that $S: C(I) \longrightarrow C^{3}(I)$ is a completely continuous linear operator. Hence, $T=S^{\circ} \mathrm{F}: \mathrm{C}^{3}(\mathrm{I}) \longrightarrow \mathrm{C}^{3}(\mathrm{I})$ is completely continuous, and the solution of BVP (1) is equivalent to the fixed point of operator $T$.

Consider homotopy family equation (25), and let $u \in C^{3}(I)$ be the solution of equation (25) corresponding to $\lambda \in(0,1)$, denote $h=\lambda F(u)$, then $u=S h \in C^{4}(I)$ is the unique solution of LBVP (5), so it satisfies (26). Multiplying both sides of equation (26) by $-u^{\prime \prime}(t)$ and combining with (H4), one can obtain

$$
\begin{aligned}
-u^{(4)}(t) u^{\prime \prime}(t) & =-\lambda f\left(t, u(t), u^{\prime}(t), u^{\prime \prime}(t), u^{\prime \prime \prime}(t)\right) u^{\prime \prime}(t) \\
& \leq \lambda\left(b_{0} u^{2}(t)+b_{1}\left(u^{\prime}(t)\right)^{2}+b_{2}\left(u^{\prime \prime}(t)\right)^{2}+b_{3}\left(u^{\prime \prime \prime}(t)\right)^{2}+\tilde{b}\right) \\
& \leq b_{0} u^{2}(t)+b_{1}\left(u^{\prime}(t)\right)^{2}+b_{2}\left(u^{\prime \prime}(t)\right)^{2}+b_{3}\left(u^{\prime \prime \prime}(t)\right)^{2}+\tilde{b} .
\end{aligned}
$$


Integrating both sides of the above inequality on $I$, together with Lemma 2 (c),

$$
\begin{aligned}
\left\|u^{\prime \prime \prime}\right\|_{2}^{2}+u^{\prime \prime}(0) u^{\prime \prime \prime}(0) & \leq \sum_{i=0}^{3} b_{i} \int_{0}^{1}\left(u^{(i)}(t)\right)^{2} \mathrm{~d} t+\widetilde{b} \\
& =\sum_{i=0}^{3} b_{i}\left\|u^{(i)}\right\|_{2}^{2}+\widetilde{b} \leq\left(\frac{b_{0}}{8}+\frac{b_{1}}{4}+\frac{b_{2}}{2}+b_{3}\right)\left\|u^{\prime \prime \prime}\right\|_{2}^{2}+\widetilde{b}
\end{aligned}
$$

By Lemma 2 (a), $\widetilde{b}-u^{\prime \prime}(0) u^{\prime \prime \prime}(0)>0$; combining with (52) and (H4),

$$
\left\|u^{\prime \prime \prime}\right\|_{2}^{2} \leq \frac{\tilde{b}-u^{\prime \prime}(0) u^{\prime \prime \prime}(0)}{\left.1-\left(\left(b_{0} / 8\right)+\left(b_{1} / 4\right)+\left(b_{2} / 2\right)+b_{3}\right)\right)}:=M_{0} .
$$

Accordingly, by Lemma 2 (c),

$$
\|u\|_{3,2}=\left(\sum_{i=0}^{3}\left\|u^{(i)}\right\|_{2}^{2}\right)^{(1 / 2)} \leq\left(\frac{1}{8}+\frac{1}{4}+\frac{1}{2}+1\right)^{(1 / 2)}\left\|u^{\prime \prime \prime}\right\|_{2} \leq \sqrt[2]{M_{0}^{(1 / 2)}} .
$$

On the basis of the boundedness of Sobolev embedding $H^{3}(I) \hookrightarrow C^{2}(I)$,

$$
\|u\|_{C^{2}} \leq c\|u\|_{3,2} \leq \sqrt{2} c M_{0}^{(1 / 2)}:=M,
$$

where $c$ is the embedding constant of $H^{3}(I) \hookrightarrow C^{2}(I)$ because

$$
\left|\lambda f\left(t, x_{0}, x_{1}, x_{2}, x_{3}\right)\right| \leq\left|f\left(t, x_{0}, x_{1}, x_{2}, x_{3}\right)\right| \leq H_{M}\left(\left|u^{\prime \prime \prime}(t)\right|\right) \text {. }
$$

Hence, it can be deduced from Lemma 4 that there exists $M_{1}=M_{1}(M)>0$, such that $\left\|u^{\prime \prime \prime}\right\|_{C} \leq M_{1}$. Combining with (55), we can infer that

$$
\|u\|_{C^{3}}=\max \left\{\|u\|_{C^{2}},\left\|u^{\prime \prime \prime}\right\|_{C}\right\} \leq \max \left\{M, M_{1}\right\}:=M_{2} .
$$

It shows that the solution set of equation (25) is bounded in $C^{3}(I)$. Then, by Lemma 3 , there is a fixed point of $T$ in $C^{3}(I)$, which is a solution of BVP (1).
This completes the proof of Theorem 3.

Remark 2. Theorem 3 guarantees the existence of the solutions for BVP (1) under the condition that $f\left(t, x_{0}, x_{1}, x_{2}, x_{3}\right)$ satisfies superlinear growth on $x_{0}, x_{1}, x_{2}$, and $x_{3} .\left(H^{*}\right)$ is a well-known Nagumo-type condition, which allows $H_{M}(\rho)$ to quadratical growth on $\rho$ at most, and it is used to restrict the growth of $f$ on $x_{3}$.

If $f_{x_{0}}, f_{x_{1}}, f_{x_{2}}$, and $f_{x_{3}}$ exist, then we can obtain the following existence result without the superlinear growth condition by the differential mean value theorem.

Corollary 2. Suppose that $f \in C\left(I \times \mathbb{R}^{4}, \mathbb{R}\right)$ satisfies $\left(H^{*}\right)$. If the partial derivatives $f_{x_{0}}, f_{x_{1}}, f_{x_{2}}$, and $f_{x_{3}}$ of $f\left(t, x_{0}, x_{1}, x_{2}, x_{3}\right)$ on $x_{0}, x_{1}, x_{2}$, and $x_{3}$ exist and satisfy,

(H5) there exist constants $b_{0}, b_{1}, b_{2}$, and $b_{3} \geq 0$ with $\left(b_{0} / 8\right)+\left(b_{1} / 4\right)+\left(b_{2} / 2\right)+b_{3}<1$ such that, for every $\left(t, x_{0}, x_{1}, x_{2}, x_{3}\right) \in I \times \mathbb{R}^{4}$,

$$
\frac{f_{x_{0}}^{2}}{4 b_{0}}+\frac{f_{x_{1}}^{2}}{4 b_{1}}-f_{x_{2}}+\frac{f_{x_{3}}^{2}}{4 b_{3}} \leq 0
$$

Then, BVP (1) has at least one solution.

Proof. Let $C_{0}=\max _{t \in I}|f(t, 0,0,0,0)|+1$; then, for every $\left(t, x_{0}, x_{1}, x_{2}, x_{3}\right) \in I \times \mathbb{R}^{4}$,

$$
\begin{aligned}
-f\left(t, x_{0}, x_{1}, x_{2}, x_{3}\right) x_{2}= & -\left[f\left(t, x_{0}, x_{1}, x_{2}, x_{3}\right)-f(t, 0,0,0,0)\right] x_{2}-f(t, 0,0,0,0) x_{2} \\
= & -f_{x_{0}}\left(t, \gamma_{0}, \gamma_{1}, \gamma_{2}, \gamma_{3}\right) x_{0} x_{2}-f_{x_{1}}\left(t, \gamma_{0}, \gamma_{1}, \gamma_{2}, \gamma_{3}\right) x_{1} x_{2} \\
& -f_{x_{2}}\left(t, \gamma_{0}, \gamma_{1}, \gamma_{2}, \gamma_{3}\right) x_{2}^{2}-f_{x_{3}}\left(t, \gamma_{0}, \gamma_{1}, \gamma_{2}, \gamma_{3}\right) x_{2} x_{3}-f(t, 0,0,0,0) x_{2},
\end{aligned}
$$


where $\gamma_{0}=\theta x_{0}, \gamma_{1}=\theta x_{1}, \gamma_{2}=\theta x_{2}$, and $\gamma_{3}=\theta x_{3}, \theta \in(0,1)$.

Since

$$
\begin{aligned}
& -f_{x_{i}}\left(t, \gamma_{0}, \gamma_{1}, \gamma_{2}, \gamma_{3}\right) x_{i} x_{2} \leq \frac{f_{x_{i}}^{2}\left(t, \gamma_{0}, \gamma_{1}, \gamma_{2}, \gamma_{3}\right) x_{2}^{2}}{4 b_{i}}+b_{i} x_{i}^{2}(i=0,1 \text {, and } 3) \text {; } \\
& -f(t, 0,0,0,0) x_{2} \leq C_{0}\left|x_{2}\right| \leq b_{2} x_{2}^{2}+\frac{C_{0}^{2}}{4 b_{2}} \text {. }
\end{aligned}
$$

Therefore, we can deduce that

$$
-f\left(t, x_{0}, x_{1}, x_{2}, x_{3}\right) x_{2} \leq b_{0} x_{0}^{2}+b_{1} x_{1}^{2}+b_{2} x_{2}^{2}+b_{3} x_{3}^{2}+\frac{C_{0}^{2}}{4 b_{2}}
$$

$$
\begin{aligned}
-f\left(t, x_{0}, x_{1}, x_{2}, x_{3}\right) x_{2} \leq & \left(\frac{f_{x_{0}}^{2}}{4 b_{0}}+\frac{f_{x_{1}}^{2}}{4 b_{1}}-f_{x_{2}}+\frac{f_{x_{3}}^{2}}{4 b_{3}}\right) x_{2}^{2} \\
& +b_{0} x_{0}^{2}+b_{1} x_{1}^{2}+b_{2} x_{2}^{2}+b_{3} x_{3}^{2}+\frac{C_{0}^{2}}{4 b_{2}} .
\end{aligned}
$$

Combining with (H5), we can see which implies that $(H 4)$ is established by denoting $\left(C_{0}^{2} / 4 b_{2}\right):=\widetilde{b}>0$. Consequently, by Theorem 3, BVP (1) has at least one solution.

The proof is completed.

Example 2. Consider the fully nonlinear BVP

$$
\left\{\begin{array}{l}
u^{(4)}(t)=u(t)+t^{2} u^{\prime}(t)+u^{\prime \prime 3}(t)+\frac{1}{2} u^{\prime \prime}(t) u^{\prime \prime \prime 2}(t)+\sqrt{2} e^{-t}, \quad t \in I, \\
u(0)=u^{\prime}(0)=u(1)=u^{\prime \prime}(1)=0
\end{array}\right.
$$

Denote

$$
f\left(t, x_{0}, x_{1}, x_{2}, x_{3}\right)=x_{0}+t^{2} x_{1}+x_{2}^{3}+\frac{1}{2} x_{2} x_{3}^{2}+\sqrt{2} e^{-t} .
$$

It is obvious that $f$ is quadratical growth on $x_{3}$, so $\left(H^{*}\right)$ is valid. In addition, for every $\left(t, x_{0}, x_{1}, x_{2}, x_{3}\right) \in I \times \mathbb{R}^{4}$,

$$
\begin{aligned}
-f\left(t, x_{0}, x_{1}, x_{2}, x_{3}\right) x_{2} & =-x_{0} x_{2}-t^{2} x_{1} x_{2}-x_{2}^{4}-\frac{1}{2} x_{2}^{2} x_{3}^{2}-\sqrt{2} e^{-t} x_{2} \\
& \leq\left|x_{0}\right|\left|x_{2}\right|+\left|x_{1}\right|\left|x_{2}\right|+\sqrt{2}\left|x_{2}\right| \\
& =2\left|x_{0}\right| \cdot \frac{\left|x_{2}\right|}{2}+2\left|x_{1}\right| \cdot \frac{\left|x_{2}\right|}{2}+2 \cdot \frac{\left|x_{2}\right|}{\sqrt{2}} \\
& \leq x_{0}^{2}+x_{1}^{2}+x_{2}^{2}+1 .
\end{aligned}
$$

Set $b_{0}=b_{1}=b_{2}=1, b_{3}=0$, and $\tilde{b}=1$, then $\left(b_{0} / 8\right)+$ $-f\left(t, x_{0}, x_{1}, x_{2}, x_{3}\right) x_{2} \leq b_{0} x_{0}^{2}+b_{1} x_{1}^{2}+b_{2} x_{2}^{2}+b_{3} x_{3}^{2}+\tilde{b}$, 
which implies that $f$ satisfies the condition ( $H 4)$, and then, by Theorem 3, BVP (63) has at least one solution.

\section{Data Availability}

The data used to support the findings of this study are included within the article.

\section{Conflicts of Interest}

The authors declare that there are no conflicts of interest regarding the publication of this paper.

\section{Authors' Contributions}

M. Wei completed the proof of the main results and the writing of the first draft. Y. Li revised the first draft and put forward some suggestions for revision. All authors read and approved the final manuscript.

\section{Acknowledgments}

This work was supported by NNSF of China (nos. 11661071 and 12061062) and NSF of Shanxi, China (no.201901D211399).

\section{References}

[1] A. R. Aftabizadeh, "Existence and uniqueness theorems for fourth-order boundary value problems," Journal of Mathematical Analysis and Applications, vol. 116, no. 2, pp. 415-426, 1986.

[2] C. P. Gupta, "Existence and uniqueness theorems for the bending of an elastic beam equation," Applicable Analysis, vol. 26, no. 4, pp. 289-304, 1988.

[3] C. P. Gupta, "Existence and uniqueness results for the bending of an elastic beam equation at resonance," Journal of Mathematical Analysis and Applications, vol. 135, no. 1, pp. 208225, 1988.

[4] D. Guo and V. Lakshmikantham, Nonlinear Problems in Abstract Cones, Academic Press, New York, NY, USA, 1988.

[5] F. Minhós, T. Gyulov, and A. I. Santos, "Lower and upper solutions for a fully nonlinear beam equation," Nonlinear Analysis, vol. 71, pp. 281-292, 2009.

[6] J. Ehme, P. W. Eloe, and J. Henderson, "Upper and lower solution methods for fully nonlinear boundary value problems," Journal of Differential Equations, vol. 180, pp. 51-64, 2001.

[7] K. Deimling, Nonlinear Functional Analysis, Springer, New York, NY, USA, 1985.

[8] M. B. M. Elgindi and Z. Guan, "On the global solvability of a class of fourth-order nonlinear boundary value problems," International Journal of Mathematics and Mathematical Sciences, vol. 20, no. 2, pp. 257-261, 1997.

[9] M. Wei and Q. Li, "Monotone iterative technique for a class of slanted cantilever beam equations," Mathematical Problems and Engineering, vol. 2017, Article ID 5707623, 2017.

[10] Q. Yao, "Positive solutions of a nonlinear elastic beam equation rigidly fastened on the left and simply supported on the right," Nonlinear Analysis: Theory, Methods \& Applications, vol. 69, no. 5-6, pp. 1570-1580, 2008.
[11] Q. Yao, "Successively iterative technique of a classical elastic beam equation with carathéodory nonlinearity," Acta Applicandae Mathematicae, vol. 108, no. 2, pp. 385-394, 2009.

[12] Q. Yao, "Solvability of a class of elastic beam equations with strong Carathéodory nonlinearity," Applications of Mathematics, vol. 56, no. 6, pp. 543-555, 2011.

[13] Q. A. Dang and T. K. Q. Ngo, "Existence results and iterative method for solving the cantilever beam equation with fully nonlinear term," Nonlinear Analysis: Real World Applications, vol. 36, pp. 56-68, 2017.

[14] Q. A. Dang and T. K. Q. Ngo, "New fixed point approach for a fully nonlinear fourth order boundary value problem," Boletim da Sociedade Paranaense de Matematica, vol. 36, pp. 209-223, 2018.

[15] R. P. Agarwal, "On fourth order boundary value problems arising in beam analysis," Differential and Integral Equations, vol. 2, pp. 91-110, 1989.

[16] Y. Li and Q. Liang, "Existence results for a fully fourth-order boundary value problem," Journal of Function Spaces and Applications, vol. 2013, Article ID 641617, 2013.

[17] Y. Li, "Existence of positive solutions for the cantilever beam equations with fully nonlinear terms," Nonlinear Analysis: Real World Applications, vol. 27, pp. 221-237, 2016.

[18] Y. Li and Y. Gao, "The method of lower and upper solutions for the cantilever beam equations with fully nonlinear terms," Journal of Inequalities and Applications, vol. 136, 2019.

[19] Y. Li and X. Chen, "Solvability for fully cantilever beam equations with superlinear nonlinearities," Boundary Value Problems, vol. 2019, no. 1, p. 83, 2019.

[20] Y. Zhang and Y. Cui, "Positive solutions for two-point boundary value problems for fourth-order differential equations with fully nonlinear terms," Mathematical Problems in Engineering, vol. 2020, Article ID 8813287, , 2020.

[21] Y. Zou, "On the existence of positive solutions for a fourthorder boundary value problem," Journal of Function Spaces, vol. 2017, Article ID 4946198, , 2017.

[22] Z. Bai, "The upper and lower solution method for some fourth-order boundary value problems," Nonlinear Analysis: Theory, Methods \& Applications, vol. 67, no. 6, pp. 1704-1709, 2007. 